\title{
Using an interactive water bottle to target fluid adherence in pediatric kidney transplant recipients: A pilot study
}

Kullgren KA, Scholl P, Kidwell KM, Hmiel SP. (2015) Using an interactive water bottle to target fluid adherence in pediatric kidney transplant recipients: A pilot study. Pediatr Transplant, 19: 35-41. DOI: $10.1111 /$ petr. 12385 .

Abstract: Hydration is important post-renal transplant to maintain adequate renal perfusion and graft function. Adherence to fluid recommendations is challenging given barriers to staying hydrated. There are no studies of adherence to fluid intake recommendations following pediatric renal transplant. Through this pilot study, we sought to determine whether the use of a commercially available interactive water bottle would lead to better adherence to recommended fluid intake and improved kidney functioning posttransplant relative to standard of care. Participants included 32 youth ages $7-19 \geq 1$ month post-kidney transplant randomized to the intervention (HydraCoach ${ }^{\circledR}$ water bottle) or standard education control group. Laboratory records were reviewed for serum chemistries (Na, BUN, creatinine) at baseline and one-month follow-up, and participants recorded daily fluid intake for 28 days. Those in the intervention group were significantly more likely to meet or exceed their fluid target, but this did not translate into better kidney functioning. Participants in the intervention group largely reported satisfaction with the water bottle and were likely to continue its use. While an interactive water bottle providing real-time feedback may be a promising intervention to help pediatric kidney transplant patients meet fluid goals, it did not appear to impact kidney function.

\author{
Kristin A. Kullgren ${ }^{1}$, Penny Scholl, Kelley \\ M. Kidwell ${ }^{2}$ and S. Paul Hmiel ${ }^{3}$ \\ ${ }^{1}$ Department of Pediatrics, University of Michigan \\ School of Medicine, Ann Arbor, MI, USA, \\ ${ }^{2}$ Department of Biostatistics, School of Public \\ Health, University of Michigan, Ann Arbor, MI, USA, \\ ${ }^{3}$ Department of Pediatrics, Washington University \\ School of Medicine/St. Louis Children's Hospital, St. \\ Louis, MO, USA
}

Key words: pediatric - renal transplant - fluid adherence

Kristin Kullgren, PhD, Assistant Professor, University of Michigan Hospital and Health Systems,

Department of Pediatrics, 1924 Taubman Center, 1500 E. Medical Center Drive, SPC 5318, Ann Arbor, MI 48109-5318, USA

Tel.: 7349367967

Fax: 7349366897

E-mail: kullgren@med.umich.edu

Accepted for publication 15 October 2014
Adequate hydration is believed to be important following renal transplant in order to maintain renal perfusion. As such, staying well hydrated is one of many self-care practices required of postrenal transplant recipients. Recommendations for high levels of fluid intake are typically given post-transplant as part of what patients are expected to do to maintain the health of their graft. In the adult renal transplant population, however, there are inconsistent data supporting this practice. While one study found fluid intake to be related to better graft function in the early months post-transplant (1), another found no such benefit a year post-transplant (2). With no empirical studies either supporting or refuting

Abbreviations: BSA, body surface area; BUN, blood urea nitrogen; PI, primary investigator. the impact of fluid intake on graft function following pediatric renal transplant, the recommendation for high fluid intake remains a standard of care.

Adherence to fluid intake is understudied in the pediatric literature despite the fact that recommendations for fluid intake are thought to be an integral part of renal post-transplant management as well as in the management of other pediatric conditions such as constipation, obesity, migraine, and sickle-cell disease. Studies in other populations where fluid intake is important suggest that adhering to hydration recommendations is challenging. For example, Kuhl, Felt, and Patton (3) reported that less than half of children with retentive encopresis met their fluid goals. Results from the adult renal transplant population echo these challenges with only about one-third of adult renal transplant recipients 
being adherent to required fluid intake volumes (4). Adherence to fluid recommendations is very difficult given the high fluid volumes required post-transplant (2).

While there is a better understanding of the challenges managing immunosuppressant medication following pediatric renal transplant (5), little is known about how youth manage their recommended fluid intake goals or what barriers youth face when trying to adhere to their fluid targets. In fact, aside from medication adherence, little is known about adherence to other aspects of the pediatric post-transplant medical regimen. In adults, not feeling thirsty, forgetting to drink, and not having the preferred fluids available are reported as interfering with adherence to fluid goals (4). We can assume that youth would experience similar challenges, especially as these barriers are common in pediatric non-adherence (6). Interventions that target these and other issues related to fluid intake could be hypothesized to improve fluid adherence.

We are proposing this study to see whether the use of an interactive, commercially available water bottle $\left(\mathrm{HydraCoach}{ }^{\circledR}\right)$ improves adherence to fluid intake goals in the post-transplant pediatric population relative to current standard-ofcare education practices. It is hypothesized that the use of this interactive bottle may address some of the challenges reported by adult transplant recipients by having fluids readily available, being able to track fluid intake, and having the bottle give feedback on achievement of target fluid goals. We hypothesized that the intervention group would show better fluid intake adherence and better serum chemistries than the control group. This study has significant relevance to the care of the pediatric kidney transplant population, as adherence to posttransplant fluid intake guidelines is believed to be important for good post-transplant kidney health and graft outcomes.

\section{Methods}

Participants $>5 \mathrm{yr}$ of age $(M=13.8 \mathrm{yr}$ [s.d. $=3.4$ ], range 7-19 yr) and more than one month post-kidney transplant $(M=51.3$ months [s.d. $=45.7$; range $1-155$ months posttransplant) were recruited from the kidney transplant clinic of St. Louis Children's Hospital. Exclusion criteria included if the family did not speak English or if the child's cognitive functioning would interfere with their ability to participate. We had a targeted enrollment of 40 participants with 20 in each arm of the study based on our convenience sample of all eligible patients in our clinic; a total of 32 families participated. Eight families refused to participate with reasons given including being too busy, believing it would be too difficult to track at day care, and fear that their nurse coordinator would find out how much they were actually drinking.
Eighty-eight percent of the sample was Caucasian American and $44 \%$ were female.

Potential participants were notified of the study by an introductory letter prior to a scheduled routine clinic visit (Time 1). Informed consent was obtained from the participant's parent or caregiver and assent from the participant, after explanation of the study by the PI or a designee at the clinic visit. This study was approved by the IRB at Washington University School of Medicine.

Participants were randomized to either the intervention (HydraCoach ${ }^{\circledR}$ water bottle) or control (standard-of-care education regarding fluid intake) group using a block randomization strategy based on week of clinic visit. This allowed all patients in a given clinic week to be enrolled in one arm of the study. We chose this method to keep participants in different arms of the study from comparing information or adherence strategies that could impact study outcomes. As a result, participants were not blind to group membership.

The HydraCoach ${ }^{\circledR}$ water bottle (Fig. 1) is an interactive water bottle that calculates personal hydration needs, tracks real-time fluid consumption, and monitors fluid intake pacing through the day (www.hydracoach.com). It is commercially available and can be purchased for $<\$ 30$. The HydraCoach $^{\circledR}$ is easy to use and program via the small removable computer on the bottle. A person merely enters their weight and the bottle automatically calculates a target fluid intake goal for that person. This can also be easily adjusted manually for a higher or lower fluid target. The HydraCoach ${ }^{\circledR}$ also prompts the user to drink by continuously visually displaying the percentage consumed in either liters or ounces. It displays total amount consumed in a 24$\mathrm{h}$ period and can be easily reset every $12-24 \mathrm{~h}$ by the push of a few buttons. The display flashes after the $24-\mathrm{h}$ period to remind the user to reset it.

Participants randomized to the intervention group received the $\mathrm{HydraCoach}^{\circledR}$ water bottle after being instructed on its use, and could keep the water bottle for

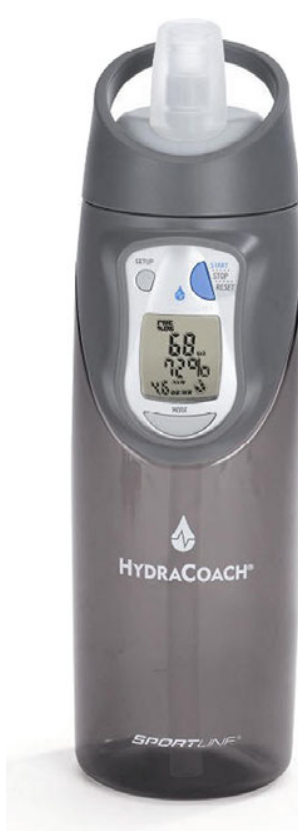

Fig. 1. HydraCoach ${ }^{\circledR}$ water bottle . 
their personal use at the end of the study. Those randomized to the standard care group (control) received the HydraCoach ${ }^{\circledR}$ water bottle for their personal use only after completion of the four-wk study. The study authors have no financial or other relationship with Hydra$\mathrm{Coach}^{\circledR}$.

All participants were asked to recall their fluid intake for the three days prior to the start of the study via a log that was included on the recruitment letter or on a form provided in clinic. After enrollment, participants were provided with a daily target fluid intake, based on their body size $\left(1500 \mathrm{~mL} / \mathrm{m}^{2} \mathrm{BSA}\right)$, and received written information regarding appropriate fluid choices. Participants randomized to the intervention group were instructed on its use after the appropriate daily target was programmed into the device by a study team member. A diary was provided for every participant to record daily fluid intake over a period of the four wk following their enrollment in the study.

After one month, when the child returned for their regularly scheduled clinic appointment, the daily diaries of each participant were collected for analysis (Time 2). All parents and youth ages 10 and above in the intervention group completed a satisfaction survey to get feedback on their perceptions of the water bottle. Participants randomized to standard-of-care control group received a HydraCoach ${ }^{\circledR}$ water bottle for their participation in the study and were instructed on its use; those who were randomized to the intervention group could keep the HydraCoach ${ }^{\circledR}$ for personal use at the end of the study.

Clinical laboratory records were reviewed for participant's serum chemistries at baseline (Time 1) and at study end (one month later-Time 2), for electrolytes (Na), BUN, and creatinine. This laboratory information was gathered as part of the patient's standard of care.

\section{Data analysis plan}

All data were analyzed using SAS v9.3. Descriptive statistics including frequencies, means, medians, and standard deviations were used to describe the sample. Age and time since transplant were compared between control and intervention groups using Wilcoxon rank-sum tests. Differences in fluid achieved from goal and sodium, BUN and creatinine levels from Time 1 to Time 2 were computed and compared using the Wilcoxon rank-sum test. These differences were also assessed between the control and intervention group controlling for time since transplant using linear regression. Logistic regression was used to assess the association between meeting or exceeding fluid target and group controlling for time since transplant.

\section{Results}

Fluid intake

There was a significant difference $(p=0.011)$ in median time since transplant between the control (5.83 yr [range 0.81-12.94]) and intervention groups (1.75 yr [range 0.08-5.96]). There was no significant difference in age between the groups ( $\mathrm{p}=0.40 ; \quad$ control group $M=14.44 \mathrm{yr}$, s.d. $=2.68$; intervention group $M=13.19 \mathrm{yr}$, s.d. $=3.92$ ). For the entire study sample, $63 \%$ tracked their fluid intake using the fluid diary. There was no significant difference in tracking by group $(\mathrm{p}=0.72)$; however, there was a lower percentage of the intervention group tracking $(56.3 \%, \mathrm{n}=9 / 16)$ vs. the control group $(68.8 \%$, $\mathrm{n}=11 / 16)$.

Participants reported fluid intake as a group ranged from $61 \%$ below target to $98 \%$ above target. There was a significant univariate difference between the difference in fluid intake at Time 2 and fluid goal between control and intervention groups $p=0.029$ (Table 1$)$. Those in the control group generally did not meet their fluid goal, with a median difference in Time 2 average fluid intake and fluid goal being -14.32 ounces (range -49.79 to 65.69 ounces) compared to those in the intervention group, who generally exceeded their fluid goal (median difference of 28.58 ounces with a range of -10.86 to 60.71 ounces). Controlling for time since transplant (which was not a significant predictor of change in fluid, $\mathrm{p}=0.86$ ), a similar trend was seen, but it is not statistically significant $(\mathrm{p}=0.077)$. Specifically, the intervention group had an expected difference in fluid intake from goal of 28.31 ounces more than the control group. This lack of significance is likely due to the small sample size and lack of power to detect such a difference (even though there are 32 participants, lack of Time 2 fluid data decreased the sample size down to 19 in the regression equation).

If we consider the dichotomous outcome of whether or not the fluid goal was reached at

Table 1. Median levels (ranges) of changes in fluid and laboratory values by group

\begin{tabular}{|c|c|c|c|c|c|}
\hline \multirow[b]{2}{*}{ Variable } & \multicolumn{2}{|c|}{ Control $(n=16)$} & \multicolumn{2}{|c|}{ Intervention $(\mathrm{n}=16)$} & \multirow[b]{2}{*}{$\mathrm{p}$-Value } \\
\hline & $n$ & Median (range) & $\mathrm{n}$ & Median (range) & \\
\hline Fluid achieved-fluid goal (oz) & 11 & $-14.32(-49.79-65.69)$ & 8 & $28.58(-10.86-60.71)$ & 0.029 \\
\hline Fluid achieved-fluid at time 1 (oz) & 6 & $-7.64(-13.77$ to -6.69$)$ & 3 & $5.50(2.69-5.81)$ & 0.028 \\
\hline Percent change in BUN & 16 & $4.56(-31.25-107.33)$ & 16 & $-2.38(-36.84-61.54)$ & 0.78 \\
\hline Percent change in sodium & 15 & $-0.72(-3.52-2.19)$ & 16 & $0(-4.86-1.45)$ & 0.29 \\
\hline Percent change in creatinine & 16 & $7.74(-16.67-44.44)$ & 16 & $8.39(-18.18-66.67)$ & 0.53 \\
\hline
\end{tabular}

p-Value is from Wilcoxon rank-sum tests without controlling for time since transplant. 
Time 2 as opposed to the exact change in fluid intake, nearly $90 \%$ of the intervention group met or exceeded their fluid goal, while $<40 \%$ of the control group reportedly met or exceeded their fluid goal. Controlling for time from transplant, the intervention group had borderline significant higher odds of reaching or exceeding their goal with an odds ratio of 2.56 (95\% CI $0.95-177.97$, $\mathrm{p}=0.055)$ and time from transplant was not significantly associated with the outcome $(p=0.91)$. If you remove time from transplant from the model, the intervention is significantly associated with the odds of meeting the fluid goal (OR 12.25, 95\% CI 1.08-138.99, $\mathrm{p}=0.043$ ). The wide confidence intervals are due to the small sample size.

\section{Laboratory outcomes}

Univariately, the percent change in BUN $(\mathrm{p}=0.78)$, sodium $(\mathrm{p}=0.29)$, and creatinine $(\mathrm{p}=0.53)$ from Time 1 to Time 2 did not differ significantly between groups. A positive percent change indicates an increase in the level from Time 1 to Time 2, whereas a negative percent change indicates a decrease in the level over time. The median percent change in BUN for the control group was $4.56 \%$ (range $-31.25 \%$ to $107.33 \%$ ) and for the intervention group $-2.38 \%$ (range -36.84 to $61.54 \%$ ). The median percent change in sodium for the control group was $-0.72 \%$ (range $-3.52 \%$ to $2.19 \%$ ) and for the intervention group was $0 \%$ (range $-4.86 \%$ to $1.45 \%$ ). The median percent change in creatinine for the control group was 7.74\% (range -16.67 to $44.44 \%$ ), and for the intervention group, the median percent change was $8.39 \%$ (range -18.18 to $66.67 \%$ ).

There was an outlier in the control group whose BUN level doubled from Time 1 to Time 2 and has the longest time from transplant to enrollment $(12.9 \mathrm{yr})$. The significance of time since transplant differs depending on including this participant or not into regression analysis for BUN, but either way, being in the intervention group was not significantly associated with percent change in BUN. There was no significant association between intervention group and percent change of sodium when controlling for time from transplant. For changes in creatinine, time from transplant was borderline significant such that a one-yr increase in time from transplant increased the expected percentage change in creatinine by $1.87 \%$, s.e. $=0.94, p=0.057$, and there was a trend for the intervention group to have higher percent change in creatinine $(12.40 \%$, s.e. $=7.07, \mathrm{p}=0.09)$.

\section{Satisfaction}

Satisfaction survey results completed by the intervention group are presented in Table 2. Results suggest that the intervention group was largely satisfied with the HydraCoach ${ }^{\circledR}$ water bottle and $67 \%$ agreed it was easy to use. However, only $50 \%$ of participants reported using the water bottle every day, $16.7 \%$ a few days, and a third reported using the water bottle only a few days. Over $50 \%$ felt that use of the water bottle improved their fluid intake. Half of the participants agreed they would continue to use the water bottle after study completion and $83 \%$ reported they felt comfortable using the water bottle around friends.

\section{Discussion}

This pilot study is the first to attempt to address the challenges of fluid adherence following pediatric renal transplant. Using a commercially available water bottle that provided feedback on intake, we hoped to alleviate some of the challenges inherent in adhering to post-transplant fluid goals. Our goal was to provide an affordable and accessible intervention that would improve fluid adherence and lead to improved serum chemistries beyond the standard-of-care clinic education. While the intervention did not

Table 2. Satisfaction with intervention $(n=12)$

\begin{tabular}{|c|c|c|c|c|c|}
\hline Item & $\begin{array}{l}\text { Strongly } \\
\text { agree }\end{array}$ & Agree & Disagree & $\begin{array}{l}\text { Strongly } \\
\text { disagree }\end{array}$ & $\begin{array}{l}\text { Don't know/ } \\
\text { blank }\end{array}$ \\
\hline The HydraCoach ${ }^{\circledR}$ water bottle was easy to use & $25.0 \%$ & $41.7 \%$ & $16.7 \%$ & $8.3 \%$ & $8.3 \%$ \\
\hline The HydraCoach ${ }^{\circledR}$ water bottle helped increase water intake & $8.3 \%$ & $50.0 \%$ & $33.3 \%$ & $8.3 \%$ & $0.0 \%$ \\
\hline $\begin{array}{l}\text { The child felt comfortable using the HydraCoach }{ }^{\circledR} \text { water } \\
\text { bottle around friends }\end{array}$ & $16.7 \%$ & $66.7 \%$ & $8.3 \%$ & $0.0 \%$ & $8.3 \%$ \\
\hline \multirow[t]{2}{*}{ The child will continue to use this bottle after the study is over } & $16.7 \%$ & $33.3 \%$ & $16.7 \%$ & $8.3 \%$ & $25.0 \%$ \\
\hline & \multicolumn{2}{|c|}{ Almost every day } & \multicolumn{2}{|c|}{ Most days } & A few days \\
\hline My child used the HydraCoach ${ }^{\circledR}$ water bottle & \multicolumn{2}{|l|}{$50.0 \%$} & \multicolumn{2}{|c|}{$16.7 \%$} & $33.3 \%$ \\
\hline
\end{tabular}


appear to significantly impact serum chemistries during the brief intervention, it remains a promising strategy that may enhance fluid adherence for some individuals post-transplant as the intervention did improve overall fluid intake relative to the individual's fluid goal. Although there was a significant difference in the time since transplant between the control and intervention group, this factor was not significantly associated with meeting or exceeding their fluid goal.

As a pilot study, we hoped to collect as much useful information as possible, from a majority of our clinic transplant population, without being unduly burdensome to participants or clinic staff. The four-wk study duration corresponded to the typical clinic and laboratory testing schedules of the participants. Block randomization of all participants in a given weekly clinic simplified participant education and training, while minimizing "cross talk" between intervention and standard care participants regarding fluid intake. With this approach, we were successful in recruiting $80 \%$ of the eligible transplant recipients. Despite several interventions to encourage recording of daily fluid intake, incomplete fluid diaries before and during the study period were a significant problem. Other study designs, such as crossover with each participant acting as their own control with and without the intervention bottle, may provide more definitive results; however, documenting daily fluid intake will likely remain as a challenge. Additionally, future researchers may consider stratification of participants based on time since transplant to minimize the impact of this important factor on outcomes.

While a few epidemiologic studies (7) suggest increased fluid intake in the general population may protect from chronic kidney disease, it remains unclear, however, whether this behavior translates into better long-term allograft function post-transplant. In the current study, participants who documented higher fluid intakes did not show any clinically significant changes in laboratory values, and in fact, a slight increase in creatinine was noted in the intervention group. Although this increase was statistically significant, it is of unclear clinical importance and may be related to the age of the graft. An elevated plasma creatinine in a kidney transplant patient may reflect acute rejection, calcineurin inhibitor (cyclosporine or tacrolimus) toxicity, or mild volume contraction with decreased renal blood flow. In the acute setting, increasing fluid intake with a normalization of creatinine can thus help exclude other, potentially more serious problems. This commonly used strategy seems most relevant in the early post-transplant months, when the risk of acute rejection is highest, and immunosuppressive regimens are changing. Our results suggest that increased oral fluid intake is a reasonable strategy and appears safe, without development of hyponatremia due to excessive fluid intake.

While serum creatinine is an accepted marker for renal function, assessing overall hydration status is more problematic. In addition to recording fluid intake, other studies have used daily urine volume, or urine specific gravity. Twentyfour-hour urine collections are inconvenient, and if recorded at home, subject to the same limitation as recording fluid intake. Urine specific gravity in a healthy individual does reflect renal free water clearance and thus overall hydration status. Although it primarily varies in response to fluid intake, however, it may be increased in response to the action of calcineurin inhibitors, or decreased in patients with chronic interstitial fibrosis, making interpretation in transplant recipients especially problematic. Only one study has examined the effects of immunosuppressive agents on renal free water clearance in transplant recipients (8), showing that free water clearance is reduced relative to healthy controls. This results in decreased urine dilution, and thus higher specific gravity, such that it is no longer an effective marker for hydration state. In addition, free water clearance decreases with loss of renal function (9), further limiting its usefulness in this population.

It was interesting to note that the intervention group tracked less than the control group. It may be that the interactive feedback from the bottle may have been enough or that it was challenging to both manage the bottle and the tracking. We also had many more participants who said they tracked than turned in their tracking forms, making it hard to determine the impact of tracking on the intervention as well as the actual intake reported. While tracking itself can be seen as an intervention in and of itself (10), whether or not participants tracked did not appear to impact their laboratory values at Time 2 or change in laboratory values over time. Ideally, a water bottle that had the ability to download data would be the best way to circumvent this issue, but unfortunately that technology is not available at this time. Many adherence interventions involve tracking as a key to success (6); tracking may have been a strong enough intervention in and of itself to offset the benefits of using the programmable bottle.

Patient satisfaction with the use of the bottle may have impacted its utility as an intervention 
tool. While most found it easy to use, this did not universally translate into actual usage, with only $50 \%$ of the group reporting using it every day during the study and a third not using at all. Given that this seemed to be a palatable intervention for about half of the study group, further information is needed on who tends to benefit from this type of intervention. It is positive that a strong majority felt comfortable using around friends as children and adolescents may disregard self-care to avoid calling attention to themselves or appearing "different" from peers (11). The benefit to using a commercially available product is that it anyone can purchase it and it does not stand out as being something different that would call attention to the child.

\section{Description of study limitations}

There are several limitations that should be taken into account while interpreting these results. Primarily, the reliance on patient self-report of fluid intake because of the bottle's inability to record data introduces possible bias and may limit the accuracy of the data. Specifically, there may have been a bias toward reporting high numbers, which may have skewed the self-report data, especially as the participants had to share their data with the clinic nurse coordinator. While keeping a diary of adherence is a very promising report strategy, families tend to overestimate adherence and only about $50 \%$ of people keep complete records (6). While our sample had a higher response rate than typically reported in the literature (6), lack of $100 \%$ response rate decreased the amount of data for analysis. Fear of sharing data with the clinic staff may have impacted response rates. The use of a small, convenience sample may have impacted our ability to detect results, particularly as our group likely included youth with and without baseline fluid adherence problems. The differences between groups are very small and therefore not detectable with the small sample size. It is also possible that the length of time in between Time 1 and 2 was not long enough for the laboratory values to change substantially. While this is the standard monitoring time for our clinic and reflects the time period one would typically monitor a recommendation to increase fluid intake, a longer Time 2 follow-up period would have likely led to more robust findings. Lack of ethnic diversity in our sample, while reflective of our clinic population during the study period, is not reflective of national trends in pediatric renal transplant and may impact the generalization of findings to other, more ethnically diverse populations (12).
Finally, while we had intended to have baseline fluid intake, we were unable to gather that data from the majority of the participants.

\section{Clinical implications}

As increasing fluid intake is often used as treatment strategy for youth with kidney transplant, using technology such as a commercially available interactive water bottle might help with adherence to those recommendations for some youth, particularly those who are interested in and enjoy using such gadgets. Use of technology has been shown to increase adherence in many studies and needs further study as these technologies grow (13). Given the difficulty directly tracking data from the bottle, it will be important to develop new technologies, such as a water bottle that can track intake wirelessly or have the ability to maintain and download data. Finally, this type of easily available technology might have applications in other populations where tracking fluid intake is important (e.g., encopresis, sicklecell disease, renal failure, heart failure, and hemodialysis).

\section{Future research}

This pilot intervention focused on the goal setting and information gathering aspects of adherence while neglecting the decision making, treatment-related action implementation, and management of the emotional aspects of adherence discussed by Creer (14). Future research should consider adding in some of these more complex aspects of fluid adherence in order to more fully intervene with this clearly challenging task. Any future research should employ gathering baseline data that would allow future interventions to better target the aspects of adherence for the individual child rather than having a onesize fits all intervention (13). As noted earlier, future research should control for and stratify time since transplant given the impact of this variable on outcomes reported here. Regardless, further exploration of fluid adherence in pediatric renal transplant is warranted.

\section{Acknowledgments}

Funding for this research was provided by the St. Louis Children's Hospital Nursing Research Grant and the University of Michigan Charles Woodson Fund for Clinical Research.

\section{Authors' contributions}

Kristin A. Kullgren: Participated in concept/design, data analysis/interpretation, drafting the article, critical revision 
of the article, and approval of the article; Penny Scholl: Participated in concept/design, drafting the article, approval of the article, securing grant funding, data collection, and approval of the article; Kelley M. Kidwell: Participated in data analyses/interpretation and approval of the article; S. Paul Hmiel: Participated in concept/design, data interpretation, critical revision of the article, and approval of the article.

\section{References}

1. Gordan EJ, Prohaska TR, Gallant M, et al. Longitudinal analysis of physical activity, fluid intake, and graft function among kidney transplant recipients. Transpl Int 2009: 22: 990998.

2. Magpantay L, Aiai F, Oberbauer R, Haas M. The effect on fluid intake on chronic kidney transplant failure: A pilot study. J Ren Nutr 2011: 21: 1-7.

3. Kuhl ES, Felt BT, Patton SR. Brief report: Adherence to fluid recommendations in children receiving treatment for retentive encopresis. J Pediatr Psychol 2009: 34: 1165-1169.

4. Gordan EJ, Prohaska TR, Gallant M, Siminoff LA. Selfcare strategies and barriers among kidney transplant recipients: A qualitative study. Chronic Illn 2009: 5: 75-91.

5. Shellmer D, Devito Dabbs A, Dew M. Medical adherence in pediatric organ transplantation: What are the next steps? Curr Opin Organ Transplant 2011: 16: 509-514.

6. RApoff MA. Adherence to Pediatric Medical Regimens, 2nd edn. NY: Springer, 2010.
7. Strippoli GF, Craig JC, Rochtchina E, Flood VM, Wang JJ, Mitchell P. Fluid and nutrient intake and risk of chronic kidney disease. Nephrology 2011: 6: 326-334.

8. Tekgunduz E, Apaydin S, Seyahi N, Altiparmak MR. Electrolyte free water clearance could be an early sign of renal dysfunction in renal transplant patients. Transplant Proc 2009: 41: 3726-3730.

9. Musso CG, Reynaldi J, Martinez B, et al. Free water clearance: Its behavior in chronic renal disease. Saudi J Kidney Dis Transpl 2011: 22: 148-150.

10. La Greca AM, Mackey ER. Adherence to pediatric treatment regimens. In: Roberts M, Steele R, eds. Handbook of Pediatric Psychology, 4th edn. New York: Guilford Press, 2009: pp. 130-152.

11. La Greca AM, Bearman KJ, Moore H. Peer relations of youth with pediatric conditions and health risks: Promoting social support and healthy lifestyles. J Dev Behav Pediatr 2002: 23: 271-280.

12. Smith JM, Martz K, Blydt-Hansen TD. Pediatric kidney transplant practice patterns and outcome benchmarks, 1987-2010: A report of the North American Pediatric Renal Trials and Collaborative Studies. Pediatr Transplant 2013: 17: 149-157.

13. Drotar D. Strategies of adherence promotion in the management of pediatric chronic conditions. J Dev Behav Pediatr 2013: 34: 716-729.

14. CREer TL. Self-management and the control of chronic pediatric illness. In: Drotar D, ed. Promoting Adherence to Medical Treatment in Chronic Childhood Illness: Concepts, Methods, and Interventions. Mahwah, NJ: Erlbaum, 2000: pp. $95-130$. 